\title{
Joint contributions of metacognition and self-beliefs to uncertainty- guided checking behavior
}

\author{
Axel Baptista ${ }^{1,2,3,4}$, Maxime Maheu ${ }^{1,5}$, Luc Mallet ${ }^{1,6,7}$, Karim N'Diaye $^{1}$
}

1. Institut du Cerveau et de la Moelle épinière, INSERM U1127, CNRS UMR7225, AP-HP PitiéSalpêtrière, Sorbonne Université, Paris, France.

2. Institut Jean Nicod, Département d'Études Cognitives, ENS, EHESS, CNRS, PSL University, Paris, France.

3. Service de Psychiatrie de l'Enfant et de l'Adolescent, GH Pitié-Salpêtrière Charles Foix, AP-HP, Paris, France.

4. Université de Paris, Paris, France.

5. University Medical Center Hamburg-Eppendorf, Hamburg, Germany.

6. Université Paris-Est Créteil, DMU IMPACT, Département Médical-Universitaire de Psychiatrie et d'Addictologie, Hôpitaux Universitaires Henri Mondor - Albert Chenevier, Assistance Publique-Hôpitaux de Paris, Créteil, France.

7. Department of Mental Health and Psychiatry, Global Health Institute, University of Geneva, Geneva, Switzerland.

Correspondence can be directed to Axel Baptista, Institut Jean Nicod, École Normale Supérieure, 29 rue d'Ulm 75005 Paris, France (e-mail: axel.baptista@gmail.com; ORCID: https://orcid.org/0000-0002-0780-5755)

\begin{abstract}
Checking behavior is a natural and adaptive strategy for resolving uncertainty in everyday situations. Here, we aimed at investigating the psychological drivers of checking and its regulation by uncertainty, in non-clinical participants and controlled experimental settings. We found that the sensitivity of participants' explicit confidence judgments to actual performance (explicit metacognition) predicted the extent to which their checking strategy was regulated by uncertainty. Yet, a more implicit measure of metacognition (derived from asking participants to opt between trials) did not contribute to the regulation of checking behavior. Meanwhile, how participants scaled on questionnaires eliciting self-beliefs such as self-confidence and self-reported obsessive-compulsive symptoms also predicted participants' uncertainty-guided checking tendencies. Altogether, these findings demonstrate that checking behavior is likely the outcome of a core explicit metacognitive process operating at the scale of single decisions, while remaining influenced by general self-beliefs. Our findings are thus consistent with two mechanisms (micro vs. macro) through which this otherwise adaptive behavior could go awry in certain psychiatric disorders such as obsessivecompulsive disorder.
\end{abstract}




\section{Introduction}

Everyday decisions are often marred by uncertainty ${ }^{1}$. Gathering new evidence through exploration is a useful and rational strategy to reduce the impact of uncertainty on subsequent decisions ${ }^{2}$. One of such exploratory strategies is checking, a procedure through which an agent repeatedly samples from the same source of information ${ }^{3,4}$. In a changing environment, checking can serve the useful purpose to update one's, possibly outdated, internal model about the world 5; for instance, checking one's smartphone for possible novel notifications. In spite of its apparent simplicity, what drives checking in humans, and how it is regulated by observer's uncertainty about the world, remains mostly unknown.

Deciphering the psychological drivers of checking is also key to understand how this, otherwise rational, behavior goes awry in certain pathological conditions such as obsessive-compulsive disorder (OCD). About $80 \%$ of OCD patients 6,7 indeed exhibit excessive checking; not only for anxiety-related, but also neutral, stimuli 8-11. In line with the hypothesized uncertainty-reducing role of checking, patients typically report that checking reduces their uncertainty about checked options ${ }^{12}$.

One hypothesis we explored here is thus that checking behavior depends on an uncertainty-evaluation, or metacognitive, process 13-17. Traditional models have decomposed metacognitive processes into two categories: (i) explicit metacognition, understood as, at least partially, conscious evaluations and regulations of our own decisions, grounded in reflective processes involving introspection, and which enables humans to share metacognitive representations with other people ${ }^{18-21}$; and (ii) implicit metacognition understood as pre-reflective, automatic evaluations and regulations of our own decisions, which does not necessarily reach awareness ${ }^{18,22}$, and which are present in non-verbal young infant 23 and non-human animals 24 . In explicit metacognition tasks, subjects are often asked to rate their confidence into some past decision ${ }^{20}$. By contrast, in implicit metacognition tasks, subjects do not explicitly rate their confidence but, instead, use it to guide decision-making, such as when choosing between several options from which to get rewarded ${ }^{25}$. In line with this taxonomy, here, we contrasted the contributions of both explicit and implicit metacognitive monitoring sensitivities to uncertainty-guided checking behavior, and explored the possibility that checking might only be related to explicit metacognition.

The propensity to check exhibits a wide spectrum from occasional to excessive forms even in the non-clinical population ${ }^{26}$. Previous studies provide converging evidence that self-beliefs (e.g. cognitive distrust) correlate with a general tendency to check, as assessed by questionnaires, in healthy subjects ${ }^{27,28}$. The mechanisms underlying this association are however still unknown. We further hypothesized that self-beliefs such as the self-reported overall propensity to compulsion and cognitive distrust, which varies across individuals, also in the non-clinical population, would impede uncertaintyguided checking behavior.

To address these questions, we capitalized on previous attempts to reproduce checking behavior in controlled experimental settings $3,4,8-10,29$. Notably, we leveraged cutting-edge experimental psychology paradigms allowing us to manipulate perceptual uncertainty through stimulus difficulty similarly across individuals, independently of overall performance. We then quantified the number of checks for different levels of perceptual uncertainty, and assessed to what extent they related to performance. We also measured the sensitivity of participants' implicit and explicit metacognition using state-of-the-art measures ${ }^{30,31}$. Finally, we made the bridge with widely used clinical 
notions by relying on validated questionnaires to assess inter-individual differences in declarative self-beliefs.

We made the following hypotheses: $\left(H_{1}\right)$ we predicted that checking reduces participants' uncertainty about checked options; $\left(H_{2}\right)$ accordingly, we expected that checking allows individuals to increase task performance: performance should be better after checking than after immediate decisions; $\left(H_{3}\right)$ importantly, we predicted that checking behavior depends on an explicit uncertainty-evaluation (explicit metacognitive accuracy) rather than an implicit uncertainty-evaluation (implicit metacognitive accuracy) process; $\left(H_{4}\right)$ finally, we expected that, above and beyond inter-individual differences in metacognitive sensitivities, participants' self-beliefs, such as self-reported compulsivity and low self-confidence, would impede checking behavior even in healthy subjects. In a nutshell, our findings show that uncertaintyguided checking behavior relates to both participants' (explicit, but not implicit) metacognitive sensitivity as well as to self-beliefs.

\section{Results}

We report results for a total of 28 human healthy participants who were asked to rate the orientation (left or right) of random dot motion (RDM) stimuli. Difficulty levels (i.e. motion coherence) were adjusted at the single participant level thanks to an initial calibration phase (see Methods), to prevent inter-individual differences in visual perception to affect the results.

Checking behavior. Participants performed a checking task in which they were allowed to replay (an infinite number of times) the RDM stimulus before committing to the decision orientation (see Methods and Figure 1a \& 1b). We first assessed fluctuations of checking behavior, how checking is regulated by stimulus difficulty and whether it improves subsequent performance.

Firstly, in order to evaluate the impact of stimulus difficulty on checking $\left(H_{1}\right)$, we used a Poisson mixed effect model explaining trial-by-trial fluctuations in the number of checks (hereafter referred to as $\mathcal{M}_{1}$; see Methods and Table S1). We found that participants increased their checking frequency for more difficult stimuli $(b=0.90, p<$ $2 \times 10^{-16}$; see Figure 1c), in line with the hypothesis that checking aims at decreasing participants' uncertainty about future decisions $\left(H_{1}\right)$.
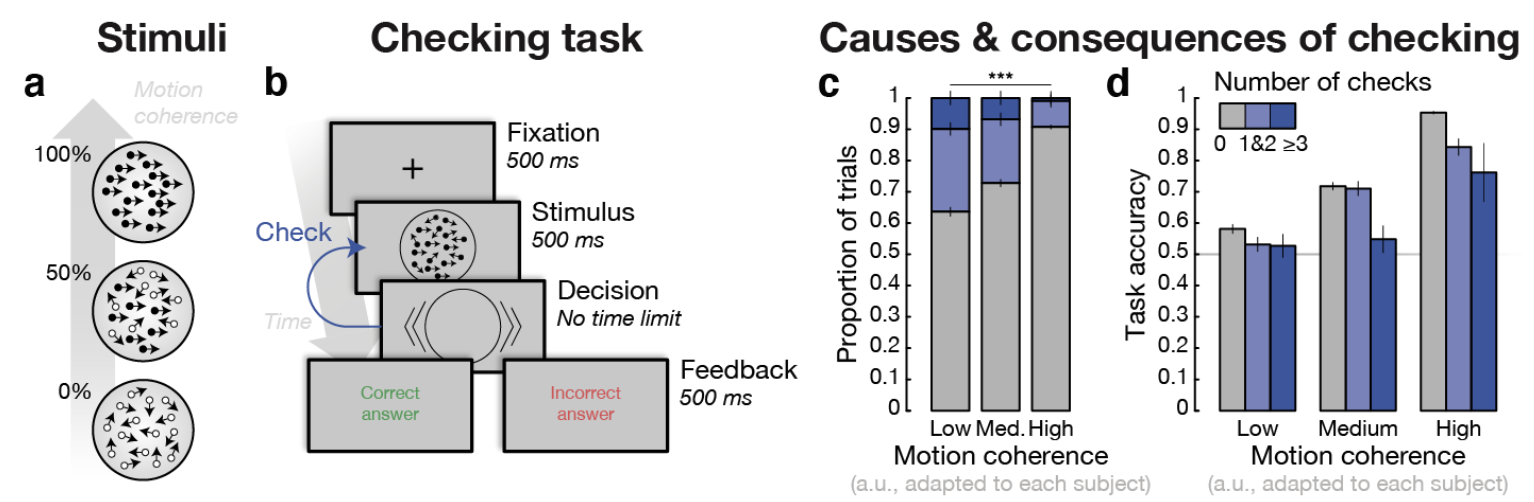

Figure 1 Causes and consequences of checking behavior a, $R$ DM stimuli. Stimuli used throughout the study were random dot motion (RDM) stimuli whose coherence level was manipulated. Participants were asked to judge the direction of motion (either left or right). $\boldsymbol{b}$, Checking task. Representative trial of the checking task: before committing to 
the decision, participants were allowed to replay the RDM stimulus an infinite number of times. Feedback was delivered at the end of the trial. c, Increased checking after more difficult stimuli. Number of checks according to motion coherence (i.e. difficulty, in arbitrary units, a.u.), which is expressed in three terciles for the sake of averaging over participants (who have different perceptual sensitivity). $d$, Decreased performance after checking. Accuracy of orientation judgments as a function of motion coherence and number of checks. Same binning method as in $\boldsymbol{c}$. Error bars correspond to standard error of the mean.

Secondly, in order to evaluate whether checking behavior improved subsequent performance $\left(\mathrm{H}_{2}\right)$, we used a multivariate logistic mixed-effect model explaining trialby-trial fluctuations in RDM task performance (hereafter referred to as $\mathcal{M}_{2}$; see Methods and Table S2). Contrary to what we expected, we found that checking decreased performance on RDM stimuli of matched difficulty levels $(b=-0.26$, $p=4.56 \times 10^{-8}$; see Figure 1d). Furthermore, we found that the detrimental effect of checking on performance decreased with stimulus difficulty (number of checks by stimulus difficulty significant and positive interaction; $b=0.20, p=6.27 \times 10^{-5}$ ): checking more strongly reduced performance for high motion coherence RDM stimuli (i.e. easy trials). Although checking was promoted by perceptual uncertainty $\left(H_{1}\right)$, it thus did not appear to reduce the impact of perceptual uncertainty on subsequent decisions $\left(H_{2}\right)$; we later discuss possible reasons for this finding (see Discussion).

Contribution of metacognition to checking. Showing that our experimental settings induced uncertainty-guided fluctuations in checking behavior, we now turn to one of our study goals: assessing the contribution of inter-individual differences in metacognitive sensitivities in the tendency of participants to check $\left(H_{3}\right)$. In particular, we assessed a possible differential effect between implicit and explicit metacognitive sensitivities in the promotion of checking and its regulation by perceptual uncertainty.

In a well-established explicit metacognition task (see for example ${ }^{32}$ ), participants were asked to explicitly rate their confidence (on a scale ranging from 1, low confidence, to 6, high confidence) about their orientation judgment (see Figure 2a). We assessed participants' explicit metacognitive sensitivity using M-ratio, a well-established measure of (explicit) metacognitive sensitivity which quantify the amount of perceptual information (in signal-to-noise units) that is available to metacognitive processes ${ }^{33}$. Overall, participants had an M-ratio significantly higher than zero (two-tailed one sample Wilcoxon signed rank test; $V$-statistic $=406 ; p=4.003 \times 10^{-6}$; see Figure $2 b$ ), thereby showing that confidence judgments were predictive of actual performance.

In an implicit metacognition task, using an original design by Barthelmé and Mamassian ${ }^{34}$ and validated in further studies ${ }^{25,30,34}$, participants had to judge the direction of two successive RDMs, of different difficulty levels (i.e. motion coherence), before being finally asked to indicate the one for which they judged to be more likely correct (see Figure 2c). We assessed participants' implicit metacognitive sensitivity by computing the difference in sigmoid slopes (relating stimulus difficulty to performance) between the discarded and the chosen stimulus (divided by the average slope across both conditions), hereafter referred to as the confidence modulation index (CMI) 25,30 . Participants' CMI was significantly higher than zero (two-tailed one sample Wilcoxon signed rank test; $V$-statistic $=351 ; p=3.814 \times 10^{-4}$; see Figure $2 d$ ), thereby showing that participants were able to leverage their metacognitive knowledge to improve decision-making. 
We then assessed the independence between implicit vs. explicit metacognitive sensitivities by correlating them across participants. We found no significant correlation $\left(r=0.04, p=0.853, \mathrm{BF}_{\text {null }}=2.83\right.$; see Figure $2 \boldsymbol{e} \&$ Table S3), thereby suggesting they reflect distinct (meta)cognitive processes and, possibly, relate differently to checking behavior. Accordingly, we also found a dissociation in the contributions of explicit vs. implicit metacognitive sensitivities to uncertainty-guided checking behavior $\left(H_{3}\right)$. On the one hand, the Poisson mixed effect model explaining trial-by-trial fluctuations in the number of checks $\left(\mathcal{M}_{1}\right)$ revealed a significant positive interaction between M-ratio and stimulus difficulty $\left(\mathcal{M}_{1} ; b=0.08, p=0.028\right.$; see Figure $\left.3 c\right)$, showing that checking was driven by perceptual uncertainty more strongly in participants with higher explicit metacognitive sensitivity. Conversely, the interaction between CMI (i.e. implicit metacognitive monitoring ability) and stimulus difficulty was negative and nonsignificant $\left(\mathcal{M}_{1} ; b=-0.05, p=0.210\right.$; see Figure $\left.3 c\right)$. We then confirmed that the differential modulation of implicit vs. explicit metacognitive sensitivities on uncertaintyguided checking behavior was significant using a likelihood ratio test (LRT) between the original model $\left(\mathcal{M}_{1}\right)$ versus a restricted model which assumes the equality between the regression coefficients of the interactions of interest (i.e. interactions between CMI and stimulus difficulty, and M-ratio and stimulus difficulty; LRT: chi-square $=5.88,1 \mathrm{df}$, $p=0.015)$. This analysis thus confirmed that checking is more strongly driven by perceptual uncertainty in participants with elevated explicit, but not implicit, metacognitive monitoring ability $\left(H_{3}\right)$.

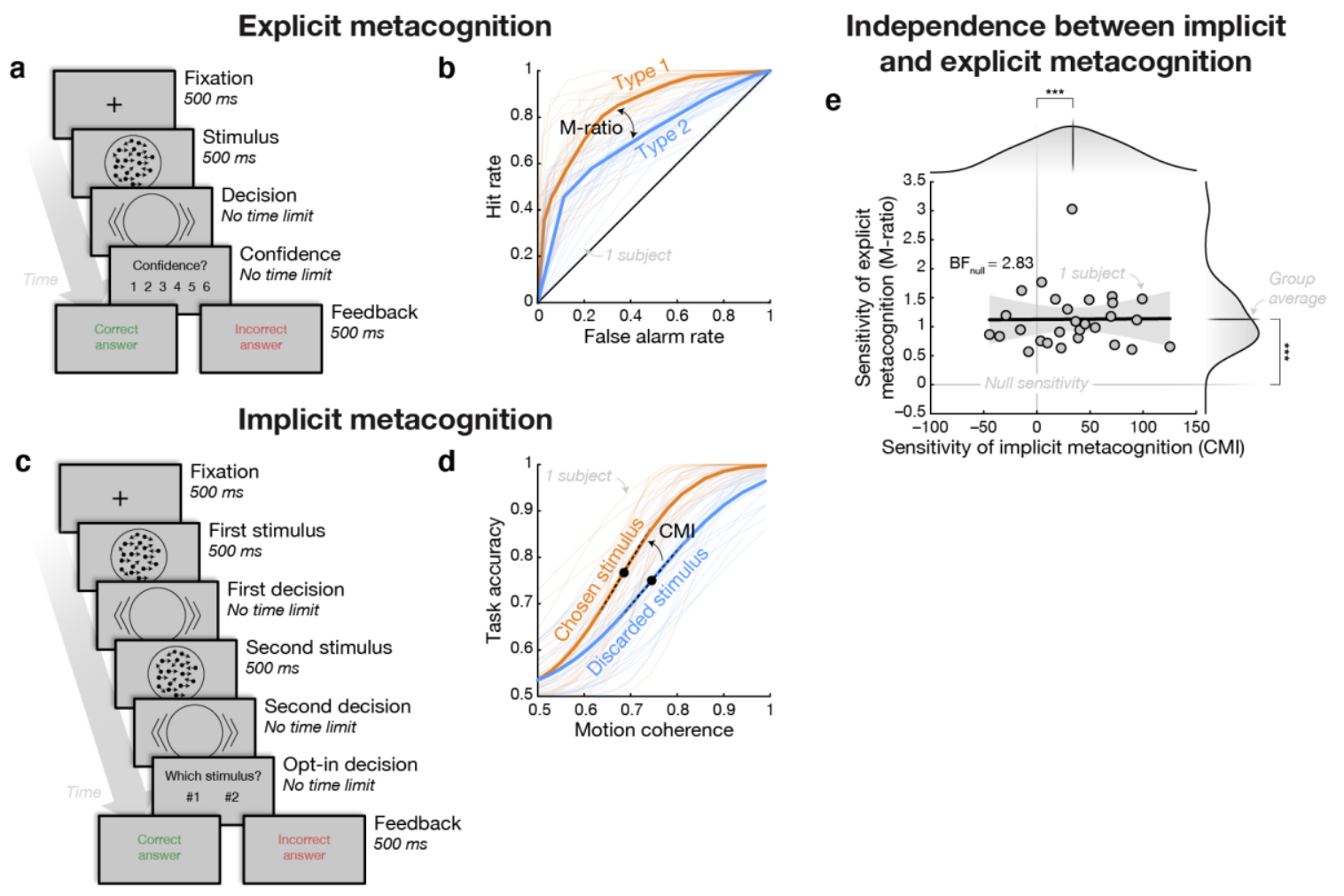

Figure 2 Independence of implicit and explicit metacognitive sensitivities a, Explicit metacognition task. Representative trial of the explicit metacognition task: after reporting their orientation judgment, participants were asked to report their confidence in this orientation judgment on a scale from 1 (guess) to 6 (certainty). Feedback was delivered at the end of each trial. $\boldsymbol{b}$, Sensitivity of explicit metacognition. The sensitivity of explicit metacognition was measured using the M-ratio, which quantifies the amount of decision information available to the confidence judgment, here depicted as the difference between type 
1 (decision) and type 2 (confidence) ROC curves ${ }^{35}$. c, Implicit metacognitive task. Representative trial of the implicit metacognition task: after reporting their orientation judgment for two successive RDM stimuli, participants were asked to report the stimulus for which they judged more likely to be correct. Feedback on the chosen stimulus was delivered at the end of each trial. $d$, Sensitivity of implicit metacognition. The sensitivity of implicit metacognition was measured as the difference in slopes between psychometric functions corresponding to the chosen minus discarded stimuli, divided by the average slope across both conditions; a measure we refer to as $\mathrm{CMI}{ }^{25,30}$. e, Independence between explicit and implicit metacognition. Metacognitive sensitivities were estimated for each participant separately in the explicit and implicit metacognition tasks. Measures are not correlated across participants suggesting they relate to independent metacognitive processes (see Table S3). Stars indicate significance: * for $p<0.05,{ }^{* *}$ for $p<0.01$, and ${ }^{* * *}$ for $p<0.001$.

Contribution of self-beliefs to checking. We hypothesized that, above and beyond inter-individual differences in metacognitive sensitivities, participants' self-beliefs, such as self-reported compulsivity and self-confidence, would modulate checking behavior even in our non-clinical population $\left(\mathrm{H}_{4}\right)$. To investigate this, we asked participants to answer questionnaires at the end of our experiment.

First, we measured the declarative lack of confidence in various cognitive domains (e.g. "my memory can mislead me at times") using the cognitive mistrust subscale of the Metacognition Questionnaire ${ }^{28}$ (see Figure $3 \mathbf{b}$ ). This subscale was of particular interest here as a long series of studies (see ${ }^{27}$ for a review) consistently showed that persons suffering from compulsive checking typically exhibit higher levels of cognitive mistrust. Here, we tested, in particular, the hypothesis that cognitive mistrust may decrease the sensitivity of checking behavior to perceptual uncertainty. Accordingly, we found that cognitive mistrust significantly reduced the effect of stimulus difficulty on checking behavior ( $\mathcal{M}_{1}$; cognitive mistrust by stimulus difficulty significant negative interaction; $b=-0.09, p=0.039$; see Figure 3c).

Second, we measured self-reported obsessive-compulsive symptoms (OCS) using the Padua inventory scale ${ }^{36}$ which quantifies self-reported obsessions and rituals across four dimensions: mental control, checking, contamination obsessions and anxiety about motor control (see Figure 3a). The Padua Inventory scale was slightly correlated with the cognitive mistrust subscale across participants $(r=0.42, p=0.026$; see Table S3), as previously observed ${ }^{28}$. We found, however, that, contrary to the cognitive mistrust subscale, the self-reported obsessive-compulsive symptoms increased the effect of stimulus difficulty on checking behavior ( $\mathcal{M}_{1}$; Padua inventory by stimulus difficulty significant positive interaction; $b=0.10, p=0.019$; see Figure $3 c$ ). 


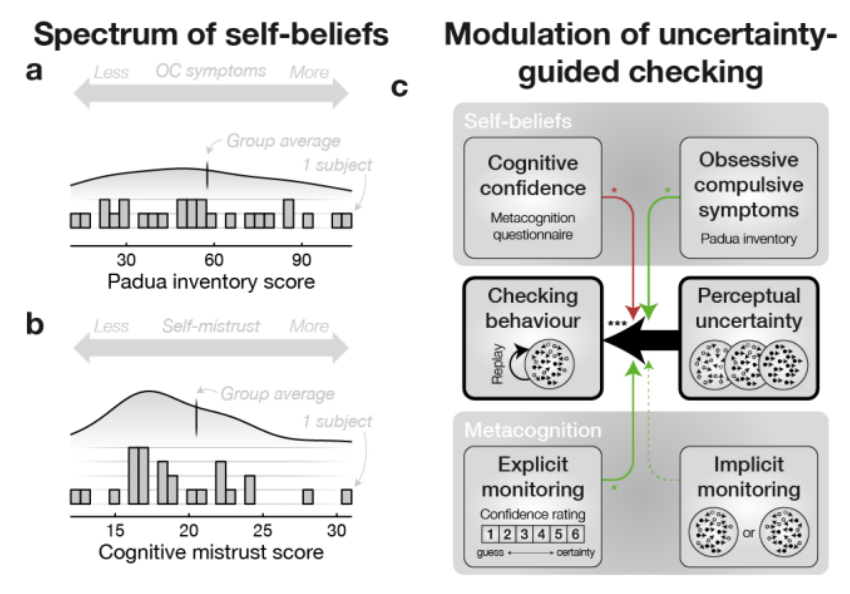

Figure 3 | Modulation of checking uncertainty-guided behavior.

$a / b$, Distribution of participants on the Padua inventory/cognitive mistrust subscales. Participants spread over a large spectrum of self-beliefs, which replicated previous findings 28,36,37 (see Methods). c, Modulation of uncertainty-guided checking. Arrow size are proportional to $z$-scores from the Poisson model of the number of checks ( $\mathcal{M}_{1}$, see Methods). Arrow color denotes the direction of the effect (green: positive, red: negative). Stars indicate significance: ${ }^{*}$ for $p<0.05$, ${ }^{* *}$ for $p<0.01$, and ${ }^{* * *}$ for $p<0.001$.

\section{Discussion}

The aim of the present study was to investigate the psychological drivers of checking behavior in humans, in particular its regulation by environmental uncertainty. Our results provide evidence that human checking behavior is the outcome of an uncertainty-evaluation, or metacognitive, process $\left(H_{1}\right)$. Indeed, participants increased their checking frequency for more difficult stimuli, and thus more uncertain settings ${ }^{38}$. This result is in line with past findings in non-human primates 4,39 which demonstrate that checking adaptively aims at decreasing one's uncertainty about future decisions.

Contrary to what was expected, checking had a detrimental effect on performance: accuracy was worse after checking than after immediate decisions $\left(H_{2}\right)$. A first possible explanation of this unexpected result relates to the psychophysical properties of the RDM stimuli used in this study: although the difficulty of the RDM stimulus was kept constant across all replays (if any), each replayed RDM differed in the exact spatial locations of the dots. A possibility is that participants expected to see the exact same RDMs across all replays. The incongruence of replayed RDMs with subject's expectations may have impaired subsequent decision-making processes. To investigate this hypothesis, future studies could manipulate the extent to which stimulus replays vary from the original stimulus and compare the impact on performance. Worth noting, above a given degree of stimulus dissimilarity, it is likely that the cognitive process at stake will relate more to 'information sampling' than genuine 'checking behavior' ${ }^{11}$. Further theoretical work is highly needed to devise the conceptual delineations of those processes. A second, perhaps more interesting, explanation for the detrimental effect of checking on subsequent performance is that repeated checking of the RDM stimuli may hinder (perceptual) decision-making processes. This possibility echoes the results of a recent meta-analysis which revealed that repeated checking reduced memory accuracy of visual stimuli across experiments, in addition to the well-known reduction in memory confidence ${ }^{40}$. The mechanisms underlying this reduction in memory performance are not yet known. A possibility is that, in our study, the diminished perceptual decision-making performance relies on similar mechanisms. Further studies are necessary to replicate our unexpected result, and to investigate its possible underpinnings. Importantly, our results show that although detrimental on average, checking nevertheless remains adaptive in the sense 
that this detrimental effect seems to decrease with stimulus difficulty, thereby suggesting it is still more adapted to check after more difficult stimuli.

Our study also provides evidence that implicit and explicit metacognitive sensitivities are dissociable $\left(H_{3}\right)$ and that they relate differently to uncertainty-guided checking behavior. We found that, in the non-clinical population, uncertainty-guided checking behavior is promoted by a conscious, explicit high-level mental representation ${ }^{18-20}$, rather than by an unconscious, implicit low-level representation of perceptual uncertainty ${ }^{18,22}$. The role of this dichotomy in shaping checking behavior has received surprisingly little attention despite its important implications for disorders of checking behavior, including OCD. It has been suggested that excessive checking behavior in compulsive checkers corresponds to the pathological automatization of an otherwise deliberate behavior $40-43$. One could thus hypothesize that such an automatization relates to an insufficient contribution of explicit metacognitive processes to uncertaintydriven checking in compulsive checkers. This insufficient contribution could result from an alteration of the ability to explicitly introspect about one's decisions (an alteration of the so-called metacognitive monitoring ability) as suggested by recent studies 44,45 . Another possibility is that the ability to explicitly introspect about one's decisions is preserved, but the resulting information is not appropriately used for the regulation of checking behavior. To our knowledge such a metacognitive control impairment has never been investigated in OCD. In either case, compulsive checkers would lack an explicit, conscious termination cue, and would continue checking regardless of environmental uncertainty.

Over and above the contribution of core metacognitive processes operating at the scale of single decisions, our results suggest that uncertainty-guided checking behavior is also influenced by self-beliefs $\left(\mathrm{H}_{4}\right)$, including the self-reported propensity to compulsions and cognitive mistrust. Previous studies provide converging evidence that self-reported overall confidence in memory is lowered by repetitive checking in healthy subjects ${ }^{40}$ and that OCD patients have lowered confidence in memory, as well as in other cognitive domains ${ }^{27}$. Nonetheless, the mechanism underlying the typical association between excessive checking and cognitive mistrust is still unknown. Our results bring a step forward in understanding by showing that cognitive mistrust impedes the regulation of checking behavior by perceptual uncertainty. It follows that the alteration of uncertainty-guided checking behavior may mediate the association between cognitive mistrust and obsessive-compulsive symptoms (OCS), a hypothesis which remains to be tested.

Conversely, our results show that beyond cognitive mistrust, self-reported OCS are associated with checking behavior better tuned to environmental uncertainty, operationalised here as stimulus difficulty. This goes against our hypothesis that the propensity to compulsion, conceived as a continuum from non-clinical OCS to OCD ${ }^{26}$, would impede uncertainty-guided checking behavior $\left(H_{4}\right)$. A possible explanation for this result is that the relation of compulsivity to uncertainty-driven checking behavior may depend on whether compulsivity falls within the clinical or non-clinical domain. The association between compulsivity and the impairment of uncertainty-guided checking behavior would thus appear clearly only for pathological compulsivity. Future studies in patients with OCD are necessary in order to test these hypotheses. To that regard, our well-controlled experimental setting might be relevant in assessing checking behavior in OCD patients.

Our findings should be relativized given the exploratory nature of our study and its limitations. In particular, we found two unexpected findings which we call for 
replications: a detrimental effect of checking on performance, and an association between increased self-reported OCS and improved uncertainty-guided checking behavior. A classic but major limitation of our study concerns our sample size. Although we collected a large number of trials to maximize within-subject reliability and sensitivity of our results, we believe it is important to confirm our results in larger sample sizes. We also note that given recent evidence reporting an impact of feedback onto self-reported measures ${ }^{46}$, it is possible that feedback provided in the course of the experiment may have influenced subsequent self-reports, including cognitive distrust and OCS. However, because performance was individually tailored (to $~ 70 \%$ accuracy), it is likely that the impact of task feedback onto subjects' self-beliefs, if any, was uniform across subjects, and thereby could not by itself explain our results. In addition, in previous research, implicit metacognition is assessed in different ways and the ideal behavioural task for its assessment is still debated. Some tasks require subjects to place bets on their decisions or give subjects the possibility to opt out ${ }^{47}$. However, whether these implicit (non-verbal) uncertainty-monitoring tasks are metacognitive is a matter of debate ${ }^{21,48}$. In the present study, the implicit metacognition task required subjects to choose among two stimuli the one they judged the most likely to yield a correct response 25,30,34. As Mamassian and colleagues showed, when doing so, participants' have to compare two levels of uncertainty related to two successive decisions ${ }^{34}$. This 'comparison step' makes the so-called implicit metacognition slightly more complex, possibly involving meta-representations, than the report of a single perceptual uncertainty quantity, which could be explained by entirely first-order processes ${ }^{48}$. Also, it is possible that in individuals' everyday environment, in particular at home, the metacognitive processes involved in repetitive checking may differ from those elicited in the lab. Accordingly, it has been shown that personalized cues trigger increased responses in OCD, compared to non-specific ones ${ }^{49}$. Our hypotheses should thus also be tested in a more ecological environment, such as at participants' homes. The development of information and communication technologies, and their recruitment for cognitive tasks, could make it possible to export research outside the laboratory and improve external validity of cognitive models.

In summary, our findings provide evidence for joint contributions of both explicit, but not implicit, metacognitive processes, and self-beliefs, including cognitive mistrust, to checking behavior guided by perceptual uncertainty. Our findings also open unexplored avenues to address and better understand how the otherwise adaptive checking behavior could go awry in OCD.

\section{Acknowledgments}

A.B. was supported by a fellowship from the Agence Régionale de Santé Ille-deFrance.

M.M. was supported by the Fondation Bettencourt Schueller.

K.N. was supported by the European Research Area Network (ERA-NET) NEURON (JTC 2013 Grant "TYMON"; Coord. L. Mallet) and Fondation pour la Recherche Médicale (FRM grant: "TORPIDO" DPP20151033971; Coord: L. Mallet).

The authors wish to thank Maxim Frolov and the Laboratoire d'Economie Expérimentale de Paris (LEEP) for helping us recruit participants.

This is a preprint of an article published in Scientific Reports. The final authenticated version is available online at: https://doi.org/10.1038/s41598-021-97958-1 


\section{Open data}

All data from this study have been made publicly available via the Open Science Framework and can be accessed at https://doi.org/10.17605/OSF.IO/2TAC4.

\section{Author contribution}

Conceptualization: A.B., M.M., K.N.; Methodology: A.B., M.M., K.N.; Software: A.B., M.M., K.N.; Investigation: A.B.; Formal analysis: A.B.; Resources: L.M., K.N.; Data Curation: A.B.; Writing - original draft: A.B., M.M.; Writing - review and editing: A.B., M.M., L.M., K.N.; Visualization: A.B., M.M.; Supervision: K.N., L.M.; Project administration: A.B., K.N.; Funding acquisition: A.B., L.M., K.N.

\section{References}

1. Bach, D. R. \& Dolan, R. J. Knowing how much you don't know: a neural organization of uncertainty estimates. Nature reviews neuroscience 13, 572-586 (2012).

2. Quilodran, R., Rothe, M. \& Procyk, E. Behavioral shifts and action valuation in the anterior cingulate cortex. Neuron 57, 314-325 (2008).

3. Hampton, R. R., Zivin, A. \& Murray, E. A. Rhesus monkeys (Macaca mulatta) discriminate between knowing and not knowing and collect information as needed before acting. Animal cognition 7, 239-246 (2004).

4. Stoll, F. M., Fontanier, V. \& Procyk, E. Specific frontal neural dynamics contribute to decisions to check. Nat Commun 7, 11990 (2016).

5. Friston, K. The free-energy principle: a unified brain theory? Nature reviews neuroscience 11, 127-138 (2010).

6. Pauls, D. L., Abramovitch, A., Rauch, S. L. \& Geller, D. A. Obsessivecompulsive disorder: an integrative genetic and neurobiological perspective. Nature Reviews Neuroscience 15, 410-424 (2014).

7. Ruscio, A. M., Stein, D. J., Chiu, W. T. \& Kessler, R. C. The epidemiology of obsessive-compulsive disorder in the National Comorbidity Survey Replication. Molecular psychiatry 15, 53-63 (2010).

8. Burbaud, P. et al. Neuronal activity correlated with checking behaviour in the subthalamic nucleus of patients with obsessive-compulsive disorder. Brain 136, 304317 (2013).

9. Clair, A.-H. et al. Excessive checking for non-anxiogenic stimuli in obsessivecompulsive disorder. European Psychiatry 28, 507-513 (2013).

10. Rotge, J. Y. et al. A challenging task for assessment of checking behaviors in obsessive-compulsive disorder. Acta Psychiatrica Scandinavica 117, 465-473 (2008).

11. Strauss, A. Y. et al. Why check? A meta-analysis of checking in obsessivecompulsive disorder: Threat vs. distrust of senses. Clinical Psychology Review 75, 101807 (2020).

12. Rachman, S. A cognitive theory of compulsive checking. Behaviour Research and Therapy 40, 625-639 (2002). 
13. Fisher, P. L. \& Wells, A. Experimental modification of beliefs in obsessivecompulsive disorder: a test of the metacognitive model. Behav Res Ther 43, 821-829 (2005).

14. Myers, S. G. \& Wells, A. Obsessive-compulsive symptoms: the contribution of metacognitions and responsibility. J Anxiety Disord 19, 806-817 (2005).

15. Myers, Samuel G., and Adrian Wells. 2013. "An Experimental Manipulation of Metacognition: A Test of the Metacognitive Model of Obsessive-Compulsive Symptoms." Behaviour Research and Therapy 51 (4-5): 177-184.

16. Myers, S. G., Fisher, P. L. \& Wells, A. Metacognition and cognition as predictors of obsessive-compulsive symptoms: A prospective study. International Journal of Cognitive Therapy 2, 132-142 (2009).

17. Tosun, A. \& Irak, M. Adaptation, Validity, and Reliability of the Metacognition Questionnaire-30 for the Turkish Population, and its Relationship to Anxiety and Obsessive-Compulsive Symptoms. Turkish Journal of Psychiatry 19, 12 (2008).

18. Shea, N. et al. Supra-personal cognitive control and metacognition. Trends in Cognitive Sciences 18, 186-193 (2014).

19. Bahrami, B. et al. Optimally Interacting Minds. Science 329, 1081-1085 (2010).

20. Fleming, S. M., Weil, R. S., Nagy, Z., Dolan, R. J. \& Rees, G. Relating Introspective Accuracy to Individual Differences in Brain Structure. Science 329, 15411543 (2010).

21. Nicholson, T., Williams, D. M., Grainger, C., Lind, S. E. \& Carruthers, P. Relationships between implicit and explicit uncertainty monitoring and mindreading: Evidence from autism spectrum disorder. Consciousness and Cognition 70, 11-24 (2019).

22. Charles, L., King, J.-R. \& Dehaene, S. Decoding the Dynamics of Action, Intention, and Error Detection for Conscious and Subliminal Stimuli. Journal of Neuroscience 34, 1158-1170 (2014).

23. Goupil, L. \& Kouider, S. Developing a Reflective Mind: From Core Metacognition to Explicit Self-Reflection. Curr Dir Psychol Sci 28, 403-408 (2019).

24. Beran, M. J. Animal metacognition: A decade of progress, problems, and the development of new prospects. Animal Behavior and Cognition 6, 223-229 (2019).

25. de Gardelle, V., Le Corre, F. \& Mamassian, P. Confidence as a Common Currency between Vision and Audition. PLOS ONE 11, e0147901 (2016).

26. Radomsky, A. S., Dugas, M. J., Alcolado, G. M. \& Lavoie, S. L. When more is less: Doubt, repetition, memory, metamemory, and compulsive checking in OCD. Behaviour Research and Therapy 59, 30-39 (2014).

27. Hermans, D. et al. Cognitive confidence in obsessive-compulsive disorder: Distrusting perception, attention and memory. Behaviour Research and Therapy 46, 98-113 (2008).

28. Larøi, F., Van der Linden, M. \& d'Acremont, M. Validity and reliability of a French version of the metacognitions questionnaire in a nonclinical population. Swiss Journal of Psychology 68, 125-132 (2009).

29. Banca, P. et al. Evidence Accumulation in Obsessive-Compulsive Disorder: the 
Role of Uncertainty and Monetary Reward on Perceptual Decision-Making Thresholds. Neuropsychopharmacology 40, 1192-1202 (2014).

30. de Gardelle, V. \& Mamassian, P. Does Confidence Use a Common Currency Across Two Visual Tasks? Psychological Science 25, 1286-1288 (2014).

31. Fleming, S. M. \& Lau, H. C. How to measure metacognition. Frontiers in Human Neuroscience 8, (2014).

32. Bang, D. \& Fleming, S. M. Distinct encoding of decision confidence in human medial prefrontal cortex. PNAS 115, 6082-6087 (2018).

33. Maniscalco, B. \& Lau, H. A signal detection theoretic approach for estimating metacognitive sensitivity from confidence ratings. Consciousness and Cognition 21, 422-430 (2012).

34. Barthelmé, S. \& Mamassian, P. Evaluation of objective uncertainty in the visual system. PLoS Comput Biol 5, e1000504 (2009).

25. Maniscalco, Brian, and Hakwan Lau. 2014. "Signal Detection Theory Analysis of Type 1 and Type 2 Data: Meta-D', Response-Specific Meta-D', and the Unequal Variance SDT Model." In The Cognitive Neuroscience of Metacognition, edited by Stephen M. Fleming and Christopher D. Frith, 25-66. Berlin, Heidelberg: Springer Berlin Heidelberg. https://doi.org/10.1007/978-3-642-45190-4_3.

36. Sanavio, E. Obsessions and compulsions: the Padua Inventory. Behaviour research and therapy 26, 169-177 (1988).

37. Cartwright-Hatton, S. \& Wells, A. Beliefs about worry and intrusions: the MetaCognitions Questionnaire and its correlates. J Anxiety Disord 11, 279-296 (1997).

38. Rollwage, M. et al. Confidence drives a neural confirmation bias. Nat Commun 11, 2634 (2020).

39. Bosc, M. et al. Checking behavior in rhesus monkeys is related to anxiety and frontal activity. Scientific Reports 7, 45267 (2017).

40. van den Hout, M. A., van Dis, E. A. M., van Woudenberg, C. \& van de Groep, I. H. OCD-like checking in the lab: A meta-analysis and improvement of an experimental paradigm. Journal of Obsessive-Compulsive and Related Disorders 20, 39-49 (2019).

41. van den Hout, M. \& Kindt, M. Repeated checking causes memory distrust. Behaviour Research and Therapy 41, 301-316 (2003).

42. Dek, E. C. P., van den Hout, M. A., Giele, C. L. \& Engelhard, I. M. Automatization and familiarity in repeated checking. Journal of Obsessive-Compulsive and Related Disorders 3, 303-310 (2014).

43. Dek, E. C., van den Hout, M. A., Giele, C. L. \& Engelhard, I. M. Automatization and familiarity in repeated checking: A replication. Journal of Experimental Psychopathology 6, 149-167 (2015).

44. Hauser, T. U., Allen, M., Rees, G. \& Dolan, R. J. Metacognitive impairments extend perceptual decision making weaknesses in compulsivity. Scientific reports 7, 1-10 (2017).

45. Rouault, M., Seow, T., Gillan, C. M. \& Fleming, S. M. Psychiatric symptom dimensions are associated with dissociable shifts in metacognition but not task performance. Biological Psychiatry (2018) doi:10.1016/j.biopsych.2017.12.017. 
46. Rouault, M., Dayan, P. \& Fleming, S. M. Forming global estimates of selfperformance from local confidence. Nature Communications 10, (2019).

47. Kepecs, A. \& Mainen, Z. F. A computational framework for the study of confidence in humans and animals. Philosophical Transactions of the Royal Society B: Biological Sciences 367, 1322-1337 (2012).

48. van den Berg, R. et al. A common mechanism underlies changes of mind about decisions and confidence. elife 5, e12192 (2016).

49. Morgiève, M. et al. Longitudinal $\mathrm{fMRI}$ assessment of cognitive and behavioral therapy for obsessive compulsive disorder: Are there neurobiological markers of response to treatment? L'Encéphale 38, S22 (2012).

50. Gelman, A. \& Hill, J. Data analysis using regression and multilevel/hierarchical models. (Cambridge university press, 2006).

51. Westfall, J., Kenny, D. A. \& Judd, C. M. Statistical power and optimal design in experiments in which samples of participants respond to samples of stimuli. Journal of Experimental Psychology: General 143, 2020 (2014).

52. World Medical Association Declaration of Helsinki. Ethical principles for medical research involving human subjects. Bull World Health Organ 79, 373-374 (2001).

53. Newsome, W. T., Britten, K. H. \& Movshon, J. A. Neuronal correlates of a perceptual decision. Nature 341, 52-54 (1989).

54. Lappin, J. S. \& Bell, H. H. The detection of coherence in moving random-dot patterns. Vision Research 16, 161-168 (1976).

55. Daunizeau, J., Adam, V. \& Rigoux, L. VBA: A Probabilistic Treatment of Nonlinear Models for Neurobiological and Behavioural Data. PLOS Computational Biology 10, e1003441 (2014).

56. Macmillan, N. A. \& Creelman, C. D. Detection theory: a user's guide. (Lawrence Erlbaum Associates, 2005).

57. Bates, D., Mächler, M., Bolker, B. \& Walker, S. Fitting Linear Mixed-Effects Models Using Ime4. Journal of Statistical Software 67, (2015).

58. Team, R. C. R: A language and environment for statistical computing [Internet]. Vienna, Austria: R Foundation for Statistical Computing; 2014. (2015).

59. Shieh, Y.-Y. \& Fouladi, R. T. The Effect of Multicollinearity on Multilevel Modeling Parameter Estimates and Standard Errors. Educational and Psychological Measurement 63, 951-985 (2003).

60. Kock, N. \& Lynn, G. Lateral Collinearity and Misleading Results in VarianceBased SEM: An Illustration and Recommendations. JAIS 13, 546-580 (2012).

61. Harrison, X. A. Using observation-level random effects to model overdispersion in count data in ecology and evolution. PeerJ 2, e616 (2014). 


\section{Methods}

Participants. Sample size was determined a priori based on past studies of metacognitive sensitivity ${ }^{25,30}$, and constrained by participant availability. We initially aimed for a target sample size of 40 participants, and our initial sample (i.e. before outlier rejection) consisted of 37 healthy volunteers. We tested our hypothesis using multilevel regression models (see below; 50 , for which it remains difficult to perform classic power calculations, mostly because of the multiple sources of variance that must be taken into account ${ }^{51}$ ). Because the study did not concern biomedical research and no medical intervention was performed, it was, according to French law, waived from getting an approval by an external ethical committee. Yet, an internal review board, the 'Comité de pilotage du Laboratoire d'Economie Expérimentale de Paris (LEEP)', assessed and approved the nature of the study and procedures, which preserved the full anonymity of the participants with respect to experimental data collection. The study was carried out in accordance with the Declaration of Helsinki 52. All participants provided informed consent and received 15 euros for participating in the study plus a bonus reward ( $\max =5$ euros) proportional to their performance during the behavioral experiment.

Behavioral experiment. The behavioral experiment, which lasted for $1 \mathrm{~h} 30 \mathrm{~min}$, consisted in 4 behavioral tasks, all derived from the well-established random-dot motion (RDM) task ${ }^{53,54}$ (see Figure 1a). The RDM stimuli consisted of 236 white dots presented for $500 \mathrm{~ms}$. Difficulty was manipulated by varying the degree of motion coherence (i.e. the proportion of dots moving in the same direction). On each trial, the direction of the dots moving coherently was randomly set to left or right. Participants reported the perceived motion direction on a keyboard; no time limit was imposed. Feedback was delivered at the end of each trial. First, participants went through a calibration phase (see below), followed by three tasks which were all explained in advance, and after a training phase of 15 trials was completed. The implicit metacognitive task and the checking task (see below) were intermixed in blocks of 50 trials for a total duration of 50 minutes, resulting in a mean number of trials of respectively 246.4 (median $=251, \quad \mathrm{sd}=21, \quad \min =204, \quad \max =300$ ) and 218.5 ( median $=201, \mathrm{sd}=22.8, \min =201, \max =251$ ). The explicit metacognition task (see below) was presented in one single block at the end of the experiment for a total duration of 15 minutes, resulting in a mean number of trials of 113.3 (median $=117.5$; $\mathrm{sd}=17.1 ; \quad \min =79 ; \quad \max =142$ ). Data from all tasks are available at https://doi.org/10.17605/OSF.IO/2TAC4.

Calibration phase. Difficulty of RDM stimuli (i.e. motion coherence) was adjusted so as to evoke the same performance level across participants. This allowed to estimate inter-individual differences in metacognitive sensitivities independently of differences in low-level visual perception, which otherwise typically contaminate metacognitive measures ${ }^{20,31}$. For that aim, prior to the experiment, we estimated, for each participant, psychometric curves (inverse logit function) relating motion coherence levels to orientation judgment accuracy in a set of 120 RDM stimuli. During calibration, coherence levels were chosen online so as to maximally improve the model evidence of the psychometric function thanks to an adaptive variational Bayesian design optimization algorithm ${ }^{55}$. We discarded 9 participants from the analyses, for which either (i) the slope of the psychometric curve derived from the calibration task was more than 2 SE below group's mean slope, or (ii) the stimulus coherence level at the inflexion point of the psychometric curve was more than 2 SE above group average. 
Our final sample consisted of 28 participants aged 19 to 29 years old (mean $=23.6$, $\mathrm{sd}=3.0$ ).

Checking task. Participants were asked to judge the orientation of a RDM stimulus they could replay an infinite number of times. Difficulty of the first RDM stimulus was drawn at random, and kept constant for the replayed RDM stimuli (if any). Dots' spatial locations varied between successive presentations of the RDM stimulus.

Implicit metacognitive monitoring task. Participants were asked to judge the orientation of two RDM stimuli presented in succession, then asked to choose the stimulus for which they felt the most likely to be correct (see Figure 20) ${ }^{25,30,34}$. Difficulty of the two RDM stimuli were varied independently. Again, using participant-specific psychometric curves estimated during the calibration task, we chose coherence levels such that participants' performance ranged from $50 \%$ to $100 \%$ correct.

Explicit metacognitive monitoring task. Participants were asked to judge the orientation of a RDM stimulus as well as a to report a post-decisional confidence judgment 20,31 using a discrete scale ranging from 1 (guess) to 6 (certainty; see Figure 2a). Prior to the experiment, participants were instructed to use the entire length of the confidence scale. They used arrows keys to move a square indicating the selected confidence level; until they reached their desired confidence level, at which point they pressed the space bar to confirm their rating. The cursor starting point was set to a random position between 1 and 6 . Using participant-specific psychometric curves estimated during the calibration task, we chose coherence levels such that participants' performance ranged from $50 \%$ to $100 \%$ correct.

Measures of metacognitive sensitivity. Implicit metacognition. We used the methodology previously implemented by de Gardelle and colleagues ${ }^{25,30}$. We fitted two psychometric curves (inverse probit function) describing the proportion of correct responses as a function of RDM difficulty separately for chosen versus discarded stimuli. We then quantified the so-called confidence modulation index (CMI) as the difference in sensitivity (i.e. slope) between the two psychometric curves, divided by the average sensitivity across of the two psychometric curves 25,30,56.

Explicit metacognition. We used the M-ratio ${ }^{31,33,35}$, a well-established measure of metacognitive efficiency which maps the sensitivity of participants' confidence judgments about their performance to their actual objective performance, independently of task difficulty.

Questionnaires. Participants answered the 'general cognitive mistrust' and 'obsessive-compulsive symptoms' questionnaires.

Cognitive mistrust. The level of cognitive mistrust was assessed with the French version of the MetaCognition Questionnaires ${ }^{28}$. Participants' mean cognitive mistrust subscale score (mean $=20.5$, sd $=6.3$ ) was similar to $M C Q$ first validation study scores in a non-clinical population (mean $=17.9$, $\mathrm{sd}=6.0$ for male, mean $=17.8$, $\mathrm{sd}=5.4$ for female) ${ }^{37}$, as well as the MCQ French validation study scores ( mean $=19.0, \mathrm{sd}=6.3$ for male, mean $=18.4, \mathrm{sd}=5.8$ for female $)$ in a non-clinical population ${ }^{28}$.

Obsessive Compulsive Symptoms (OCS). OCS were assessed using the Padua Inventory ${ }^{36}$, a 60 -items Likert scale which measures obsessions and rituals (motor or mental). Participants' mean Padua inventory score (mean $=57.6$, $\mathrm{sd}=28.8$ ) was similar to Sanavio et al.'s study with non-clinical participants' (mean $=53.6, \mathrm{sd}=27.7$ for male, mean $=62.5$, sd $=29.2$ for female) ${ }^{36}$. 
Statistical models. Models were implemented using the Ime $4{ }^{57}$ package in $\mathrm{R}$ version 3.4.1 58. Regression coefficients $(b)$ and their statistics ( $z$-scores and $p$-values), calculated using the Wald method 57 , as well as bootstrapped $95 \%$ confidence intervals, are reported (see Table S1 \& S2). All predictors were standardized. For each regressor in the models, we checked for multicollinearity using the variance inflation factor (VIF; Shieh and Fouladi 2003). A VIF smaller than 3 indicates that collinearity is not an issue ${ }^{60}$.

Model \#1 $\left(\mathcal{M}_{1}\right)$. The model relates trial-by-trial fluctuations of the number of checks to trial-by-trial changes in RDM difficulty, as well as trait-level predictors including implicit and explicit metacognitive sensitivities (i.e. CMI and M-ratio, respectively), questionnaires (i.e. Padua Inventory scale and MCQ's cognitive mistrust subscale) as well as interactions between these predictors and trial-level stimulus difficulty. Practically, the model is a Poisson generalized linear mixed model $\left(N_{\text {obs }}=6119\right.$; $N_{\text {subj }}=28$ ) with participants as random intercept, and an observation level random effect used to account for the overdispersion of the dependent variable, as recommended by Harrison ${ }^{61}$. We computed VIF for all regressors and found an average VIF of $1.2(\mathrm{sd}=0.1$, median $=1.1$, $\max =1.4)$, demonstrating collinearity was not an issue in our model. This model allowed us to test hypotheses $H_{1}, H_{3}$, and $\mathrm{H}_{4}$.

Model \#2 $\left(\mathcal{M}_{2}\right)$. The model relates trial-by-trial fluctuations in the accuracy of orientation judgments to trial-by-trial fluctuations in number of checks, RDM difficulty as well as trait-level predictors including implicit and explicit metacognitive sensitivities (i.e. CMI and M-ratio, respectively), Padua Inventory and MCQ's cognitive mistrust subscale, as well as interactions between these predictors and trial-level number of checks. Practically, the model is a logistic linear mixed model (Nobs $=6119$; Nsubj $=28$ ) with participants as random intercept. Again, we found an average VIF of $1.13(\mathrm{sd}=0.7$, median $=1.1$, $\max =2.8)$, demonstrating collinearity was not an issue in our model. This model allowed us to test hypothesis $\mathrm{H} 2$. 


\section{Supplementary information}

Table S1 | Poisson mixed model $\left(\mathcal{M}_{1}\right)$ of the number of checks

Regression coefficients of main and first-order interaction effects.

\begin{tabular}{|c|c|c|c|c|}
\hline & $\begin{array}{c}\text { Estimate } \\
\text { (SE) }\end{array}$ & $\begin{array}{c}\text { Bootstrapped Cl } \\
{[2.5 \%, 97.5 \%]}\end{array}$ & z-value & $p$-value \\
\hline \multicolumn{5}{|l|}{ Main effects } \\
\hline Intercept & $\begin{array}{l}-2.01 \\
(0.10)\end{array}$ & {$[-2.34,-1,89]$} & -20.86 & $<2 \times 10^{-16 * * *}$ \\
\hline M-ratio & $\begin{array}{c}0.17 \\
(0.09)\end{array}$ & {$\left[-4.54 \times 10^{-3}, 0.33\right]$} & 1.95 & 0.051 \\
\hline CMI & $\begin{array}{l}0.18 \\
(0.09)\end{array}$ & {$\left[-1.22 \times 10^{-3}, 0.35\right]$} & 1.94 & 0.053 \\
\hline Cognitive mistrust & $\begin{array}{c}0.10 \\
(0.10)\end{array}$ & {$[-0.10,0.27]$} & 1.00 & 0.316 \\
\hline Padua inventory & $\begin{array}{l}-0.03 \\
(0.10)\end{array}$ & {$[-0.21,0.16]$} & -0.28 & 0.777 \\
\hline Difficulty & $\begin{array}{c}0.90 \\
(0.04)\end{array}$ & {$[0.81,0.95]$} & 23.57 & $<2 \times 10^{-16 * * *}$ \\
\hline \multicolumn{5}{|l|}{ Interaction effects } \\
\hline M-ratio $\times$ Difficulty & $\begin{array}{l}0.08 \\
(0.04)\end{array}$ & {$\left[2.27 \times 10^{-2}, 0.16\right]$} & 2.20 & 0.028 * \\
\hline CMI x Difficulty & $\begin{array}{l}-0.05 \\
(0.04)\end{array}$ & {$\left[-0.12,2.83 \times 10^{-2}\right]$} & -1.25 & 0.210 \\
\hline $\begin{array}{l}\text { Cognitive } \\
\text { mistrust } \times \text { Difficulty }\end{array}$ & $\begin{array}{l}-0.09 \\
(0.04)\end{array}$ & {$\left[-0.16,-2.41 \times 10^{-4}\right]$} & -2.06 & 0.039 * \\
\hline $\begin{array}{l}\text { Padua } \\
\text { inventory } \times \text { Difficulty }\end{array}$ & $\begin{array}{c}0.10 \\
(0.04)\end{array}$ & {$\left[1.60 \times 10^{-2}, 0.18\right]$} & 2.35 & 0.019 * \\
\hline
\end{tabular}


Table S2 | Logistic mixed regression model $\left(\mathcal{M}_{2}\right)$ of performance

Regression coefficients of main and first-order interaction effects.

\begin{tabular}{|c|c|c|c|c|}
\hline & $\begin{array}{c}\text { Estimate } \\
\text { (SE) }\end{array}$ & $\begin{array}{c}\text { Bootstrapped Cl } \\
{[2.5 \%, 97.5 \%]}\end{array}$ & z-value & $p$-value \\
\hline \multicolumn{5}{|l|}{ Main effects } \\
\hline Intercept & $\begin{array}{c}1.41 \\
(0.07) \\
\end{array}$ & {$[1.28,1.54]$} & 21.23 & $<2 \times 10^{-16 * * *}$ \\
\hline Checking & $\begin{array}{l}-0.26 \\
(0.05)\end{array}$ & {$[-0.36,-0.16]$} & -5.47 & $4.56 \times 10^{-8 * * *}$ \\
\hline Difficulty & $\begin{array}{l}-1.12 \\
(0.04)\end{array}$ & {$[-1.21,-1.04]$} & -25.59 & $<2 \times 10^{-16 * * *}$ \\
\hline M-ratio & $\begin{array}{l}-0.05 \\
(0.06)\end{array}$ & {$\left[-0.17,6.96 \times 10^{-2}\right]$} & -0.86 & 0.389 \\
\hline CMI & $\begin{array}{c}0.05 \\
(0.06) \\
\end{array}$ & {$\left[-8.32 \times 10^{-2}, 0.16\right]$} & 0.77 & 0.440 \\
\hline Padua inventory & $\begin{array}{c}0.12 \\
(0.07)\end{array}$ & {$\left[-2.23 \times 10^{-2}, 0.26\right]$} & 1.77 & 0.076 \\
\hline Cognitive mistrust & $\begin{array}{l}-0.03 \\
(0.07)\end{array}$ & {$[-0.17,0.10]$} & -0.42 & 0.672 \\
\hline \multicolumn{5}{|l|}{ Interaction effects } \\
\hline Checking × Difficulty & $\begin{array}{c}0.20 \\
(0.05)\end{array}$ & {$\left[9.09 \times 10^{-2}, 0.29\right]$} & 4.00 & $6.27 \times 10^{-5 * \star \star}$ \\
\hline Checking $\times$ M-ratio & $\begin{array}{l}0.005 \\
(0.03)\end{array}$ & {$\left[-6.19 \times 10^{-2}, 6.57 \times 10^{-2}\right]$} & 0.17 & 0.867 \\
\hline Checking $\times$ CMI & $\begin{array}{c}0.02 \\
(0.03) \\
\end{array}$ & {$\left[-4.33 \times 10^{-2}, 9.23 \times 10^{-2}\right]$} & 0.58 & 0.562 \\
\hline $\begin{array}{l}\text { Checking } \times \text { Padua } \\
\text { inventory }\end{array}$ & $\begin{array}{l}-0.03 \\
(0.03)\end{array}$ & {$\left[-9.87 \times 10^{-2}, 4.54 \times 10^{-2}\right]$} & -0.81 & 0.419 \\
\hline $\begin{array}{l}\text { Checking } \times \text { Cognitive } \\
\text { mistrust }\end{array}$ & $\begin{array}{l}-0.07 \\
(0.04)\end{array}$ & {$\left[-0.15,-3.53 \times 10^{-3}\right]$} & -2.00 & $0.045^{*}$ \\
\hline
\end{tabular}

Table S3 | Correlation matrix using spearman method with listwise deletion

\begin{tabular}{|c|c|c|c|}
\cline { 2 - 4 } \multicolumn{1}{c|}{} & CMI & $\begin{array}{c}\text { Cognitive } \\
\text { mistrust }\end{array}$ & $\begin{array}{c}\text { Padua Inventory } \\
\text { score }\end{array}$ \\
\hline M-ratio & $\begin{array}{c}r=0.04 \\
(p=0.853)\end{array}$ & $\begin{array}{c}r=0.03 \\
(p=0.880)\end{array}$ & $\begin{array}{c}r=-0.16 \\
(p=0.416)\end{array}$ \\
\hline CMI & & $\begin{array}{c}r=-0.16 \\
(p=0.408)\end{array}$ & $\begin{array}{c}r=-0.10 \\
(p=0.627)\end{array}$ \\
\hline $\begin{array}{c}\text { Cognitive } \\
\text { mistrust }\end{array}$ & & & $r=0.42$ \\
& & & $\left(p=0.026^{\star}\right)$ \\
\hline
\end{tabular}

